\title{
Aproximación al sistema sanitario militar borbónico durante la guerra de Sucesión española. El frente catalán, 1705-1714
}

\author{
Adrià CASES IBÁÑEZ \\ Grup de Recerca Manuscrits - Universidad Autónoma de Barcelona \\ adriacases@gmail.com
}

Recibido: 22 de marzo de 2011

Aceptado: 22 de enero de 2014

\begin{abstract}
RESUMEN
Durante el conflicto sucesorio español (1702-1715) fueron millares los soldados que se encontraron en los campos de batallas o en los sitios a plazas y fortalezas. Estos hombres vivían el desarrollo de la guerra con grandes carencias, situación que les condenaba a demandar cierta asistencia sanitaria. En este trabajo analizamos la respuesta que dio la estructura militar borbónica en el frente catalán. Básicamente, sacaremos a la luz los modelos de organización hospitalarios e intentaremos estudiar la atención que recibieron dichos militares. También abordaremos las distintas etapas de la contienda, factor absolutamente clave para conocer los procesos anteriormente mencionados.
\end{abstract}

Palabras clave: Guerra de Sucesión española, hospital, enfermedad, asistencia, asiento, Cataluña, Aragón.

\section{Approach to The Bourbon Military Care System During The War of The Spanish Succession. The Catalan Front, 1705-1714}

\begin{abstract}
During the War of the Spanish Succession (1702-1715) thousands of soldiers were involved not only in the battlefields but also in the besiegements that took place in different cities and fortresses. These men lived their daily routine in the battlefield through all kinds of shortages towards their basic needs which made them demand a sanitary assistance. In this research study we will analyse how the Borbonic Military Commandment operated in the Catalan front regarding this matter. Basically, our aim is to shed a light on the hospital structure at the time and how they were organized. Also, we aim to know which kind of attention these soldiers ended up having. We will also go through the different stages of the conflict which is a key factor to get to know all the processes mentioned before.
\end{abstract}

Key words: War of the Spanish Succession, hospital, disease, care, contract, Catalonia, Aragon. 


\section{Introducción $^{1}$}

Mucho se ha escrito sobre la dinastía francesa que se implantó en España a inicios del siglo XVIII. El nuevo monarca quiso cambiar el rumbo del Estado, imitando el aparato administrativo que había diseñado su abuelo Luis XIV en la centuria precedente. Para conseguirlo, Jean Orry se desplazó a Madrid, implementando el primer reformismo de corte hacendístico que marcó los sucesivos años de la contienda bélica que se avecinaba. Durante este período (1701-1705) los cambios introducidos buscaban consolidar la "vía reservada" a través de la cual los distintos despachos interactuaron con el monarca de manera directa. De esta forma, los Consejos quedaban en un segundo término. De entre los nuevos instrumentos -teniendo en cuenta nuestros objetivos-, destaca la creación de la Secretaría del Despacho de Guerra (posteriormente de Guerra y Hacienda) y asociada a ella, la Tesorería Mayor de Guerra. Uno de los objetivos principales de estas oficinas pasaba por controlar y asegurar la financiación de todo lo relativo a la milicia. Para ello, durante estos primeros años, Orry buscó inversores fuertes que pudieran proveer al ejército de los pertrechos y servicios necesarios ${ }^{2}$.

A su vez, debido a la desastrosa herencia castrense del último Habsburgo ${ }^{3}$, el reformismo administrativo apuntado se desarrolló en paralelo a un proceso de renovación total de las armas hispánicas. En esta tesitura, la Real Ordenanza del 28 de septiembre de 1704 marca el inicio del ejército borbónico de nuevo cuño. Con este reglamento (y los sucesivos) se perseguía un objetivo triple: reformar los cuerpos a nivel orgánico, controlar política y administrativamente la milicia y aplicar un nuevo paradigma social (atraer a la nobleza) ${ }^{4}$. Esta voluntad de codificación se capta perfectamente gracias al valioso trabajo de recopilación que realizó a mediados del Setecientos J. A. Portugués: según su colección de ordenanzas, entre 1701 y 1715 Felipe V promulgó 61 normativas de distinta naturaleza que reglamentaron todos los aspectos de los principales cuerpos del ejército (infantería, caballería, dragones, Consejo de Guerra, gobernadores de plazas, directores, entre otros estamentos) ${ }^{5}$.

En el presente trabajo no nos centraremos en estos dos procesos que hemos insinuado, su cobertura en términos historiográficos es sobradamente solvente. De todas

${ }^{1}$ El presente estudio es fruto de la estancia breve realizada en el Departamento de Historia Moderna de la UCM (enero-junio 2010) dentro del marco de las ayudas predoctorales del Ministerio de Ciencia e Innovación a la formación de personal investigador. Agradezco a la Dra. Virginia León Sanz su amabilidad al acogerme como tutora durante mi estancia.

2 Dubet, A.: Un estadista francés en la España de los Borbones: Juan Orry y las primeras reformas de Felipe V (1701-1706), Madrid, Biblioteca Nueva, 2008; "La nueva política crediticia de la Corona a principios del siglo XVIII: la creación del Tesorero Mayor de Guerra en España (1703-1706)", Studia Historica. Historia Moderna, 30 (2008), pp. 191-216.

3 Espino López, A.: "El declinar militar hispánico durante el reinado de Carlos II", Studia Historica. Historia Moderna, 20 (1999), pp. 173-198.

4 Andújar Castillo, F.: "La reforma militar en el reinado de Felipe V", en Pereira Iglesias, J. L. (coord.): Felipe V de Borbón 1701-1746: actas del congreso de San Fernando (Cádiz) de 27 de noviembre a 1 de diciembre de 2000, Fundación Municipal de Cultura del Ayuntamiento de San Fernando (Cádiz), Universidad de Córdoba, pp. 615-640.

5 Portugués, J. A.: Colección General de las Ordenanzas Militares. Sus innovaciones y aditamentos, tomos I-II, Madrid, Imprenta de Antonio Marín, 1764. 
formas, cabe mencionar que la práctica sanitaria militar implementada durante el conflicto sucesorio se encuentra inmersa en este telón de fondo de reformulación administrativa y orgánica. A pesar de que en los últimos años las aportaciones sobre los ejércitos y el constructor de la guerra han aumentado cualitativamente gracias a la concreción de muchos aspectos difíciles de historiar, la sanidad militar del frente es aún una incógnita generalizada. Los historiadores de la milicia que se preocupan por conocer los condicionamientos sociales de los combatientes modernos, tienen en la asistencia sanitaria un reto que puede acercarles a una cotidianidad abrumadora. Como veremos a continuación, una mezcla de inoperatividad estatal, de iniciativas privadas y, cómo no, de protección local, están en la base de esta coyuntura.

Buena parte de los estudios bélico-sanitarios de época moderna en España van estrechamente ligados a la presencia naval. En este sentido, destacamos las aportaciones de M. Gracia. También los trabajos de M. Parrilla -aunque situados en la primera modernidad- nos sirven para conocer el funcionamiento de hospitales en frentes activos, tal y como nosotros pretendemos tratar en este estudio. J. M. Massons es el impulsor de la obra más transversal sobre la sanidad militar ya que aborda estos aspectos desde los precedentes de época antigua y medieval hasta el siglo XX. Asimismo, este médico-historiador nos ha facilitado algunas notas de gran utilidad sobre los hospitales militares de Barcelona entre los siglos XV-XIX. Las acotaciones que ofrece L. White también son provechosas para hacernos una idea del tipo de tratamiento médico que tuvieron los soldados, con informaciones exactas sobre las curas empleadas en heridas y enfermedades ${ }^{6}$.

En relación a la guerra de Sucesión española, podemos destacar los trabajos que se publicaron en las actas del décimo encuentro sobre historia militar, realizado en Sevilla hace ya más de una década. Las aportaciones de M. Martínez y la saga Velamazán abrieron algún claroscuro y sobre todo, muestran el camino para conocer los fondos -aunque escasos- que contempla Simancas. De la misma forma, cabe mencionar los estudios sobre enfermedad y muerte durante la guerra de los Nueve Años, precedente indispensable de la contienda que nos ocupa. Los trabajos que nos han sido (y son) de mayor utilidad los hizo J. Riera y de hecho, el estudio que presentamos en estas páginas completa las investigaciones que el autor elaboró acerca de los asientos de hospitales militares en el siglo XVIII?

6 Gracia Rivas, M.: "Los Hospitales Reales del Ejército y Armada en las campañas militares del siglo XVI", en GARCíA HERNÁN y MAFFI (eds.): Guerra y sociedad en la monarquía hispánica: política, estrategia y cultura en la Europa Moderna (1500-1700), vol. II, Madrid, Ediciones Laberinto, Fundación Mapfre, CSIC, 2006, pp. 765-784. La Sanidad naval española. Historia y evolución, El Ferrol, Bazán, 1995. "El hospital naval de Ferrol en 1589", Revista de Historia Naval, 32 (1991), pp. 95-116. Parrilla Hermida, M.: El hospital militar español de Malinas en los siglos XVI y XVII, Madrid, 1964. MAssons I Esplugas, J. M.: Historia de la Sanidad Militar Española, 4 vols., Barcelona, Ediciones Pomares-Corredor, 1994. Véase del mismo autor "Els hospitals militars de Barcelona", Gimbernat: revista catalana d'història de la medicina i de la ciència, 9 (1988), pp. 225-235. White, L.: "Los Tercios en España: el combate", Studia Storica. Historia Moderna, 19 (1998), pp. 158-162.

7 Velamaź́n Díaz, V. y otros: "La medicina española durante la Guerra de Sucesión”, en La Guerra de Sucesión en España y América: actas X Jornadas Nacionales de Historia Militar, Sevilla, 13-17 de noviembre de 2000, Madrid, Deimos, 2001, pp. 929-942. MArtínez Cerro, M.: "La sanidad naval durante la guerra de Sucesión española (1701-1715)", en La Guerra de Sucesión en España y América: actas X Jornadas Nacionales de Historia Militar, Sevilla, 13-17 de noviembre de 2000, Madrid, Deimos, 2001, pp. 451-460. EsPINO LóPEZ, 
Así pues, a continuación intentaremos abordar una realidad que en ocasiones se desvela demoledora. A modo de ejemplo, citaremos la situación que hemos localizado en Lleida a finales de 1708, cuando el coronel del regimiento de Navarra -que estaba acantonado en dicha plaza- se quejaba a Grimaldo porque tenía la mayoría de su cuerpo incapacitado para ejercer el servicio: "se allan los soldados echos unos Adanes [...] que nosé como han de tolerar este invierno". Una protesta absolutamente razonable si tenemos en cuenta que dicho coronel, en el momento de escribirla, tenía el $62,3 \%$ de su regimiento enfermo o convaleciente ${ }^{8}$.

La rotundidad de este tipo de datos nos hace preguntarnos sobre la sanidad presente en el ejército. Centrándonos en el frente catalán (y frontera oriental aragonesa), nuestro propósito consiste en averiguar qué tipo de soluciones ofreció la Corona para paliar estas insuficiencias, dónde las promovió y el impacto que tuvo entre los soldados afectados. Para lograrlo hemos trabajado (y cruzado) distintas fuentes archivísticas. Básicamente, la documentación de Estado que alberga el Archivo Histórico Nacional, la sección suplementaria de la Secretaría de Guerra depositada en Simancas (tal y como han abordado otros autores) y los fondos del Arxiu de l'Hospital de la Santa Creu de Barcelona.

Somos conscientes que los materiales para afrontar estos aspectos con plenas garantías no son completos, lo que imposibilita reconstruir la totalidad de la situación acaecida. De todas formas, los resultados extraídos nos hacen ser optimistas y esperamos haber iniciado un camino que nos conduzca a explicar mejor uno de los procesos que influyeron más en la cotidianidad de los hombres que lucharon por el trono español.

\section{Los asientos hospitalarios}

Anteriormente hemos situado el reglamento de la Real Ordenanza de 1704 como bisagra entre los ejércitos hispánicos de los siglos XVII y XVIII. La sanidad también se vio afectada por esta nueva codificación y se reorganizaron algunos aspectos relativos al personal de los hospitales. En cada sanatorio se asignó un doctor en medicina, un cirujano mayor, un boticario, dos ayudantes de cirujano mayor, doce practicantes de cirugía y treinta acémilas (art. 124-129) ${ }^{9}$. Por una parte, el objetivo consistía en definir de manera clara la división de trabajo entre los médicos, los cirujanos y los boticarios. Por otra, las cuestiones relativas a la administración quedaban relegadas exclusivamente a los directores y los controladores, que ganaron peso en los hospitales. Durante el conflicto, el ejército borbónico contó con un cirujano por regimiento

\footnotetext{
A.: "Enfermedad y muerte en el Ejército de Cataluña durante la Guerra de los Nueve Años, 1689-1697", $D y$ namis: Acta hispanica ad medicinae scientiarumque historiam illustrandam, 16 (1996), pp. 427-444. RIERA Palmero, J.: Ordenanzas y asientos de hospitales militares en España, Valladolid, Universidad, Secretariado de Publicaciones, 1992.

8 Archivo Histórico Nacional (AHN), Estado, leg. 353. Carta de Manuel de Navarra a Grimaldo. Lleida, 13 de octubre de 1708.

9 Velamazán Díaz y otros, op. cit. (nota 7), pp. 934-935. Portugués, op. cit. (nota 5), tomo I, p. 386.
} 
que aumentó a dos a partir de $1715^{10}$. Posiblemente, la contienda puso en evidencia la insuficiencia de estas medidas y en plena guerra -como veremos más adelante-se tuvieron que promulgar nuevas directrices para mejorar la gestión de los hospitales. En cierta medida, cada período bélico, y éste no fue una excepción, fue un laboratorio para todas aquellas cuestiones que concernían al mundo sanitario castrense.

Gracias a los estudios pioneros de J. Danón sabemos que desde los primeros años de la época moderna los militares fueron atendidos en el hospital de la Santa Creu ${ }^{11}$, principal institución asistencial y hospital general de Barcelona. Sin embargo, fue sobre todo a partir del siglo XVII -y el impacto cuantitativo que significó este siglo en términos militares-, cuando el ingreso del estamento castrense se regularizó; básicamente, a través del puerto, ya que las galeras que llegaban a la Ciudad Condal siempre contribuyeron al incremento de enfermos debido a las duras condiciones del transporte marítimo. En 1638, en plena guerra contra la Francia de Luis XIII, el virrey conde de Santa Coloma emitió una orden donde se verifica la necesidad económica que sufría la institución y a su vez, la presencia militar en la misma: "attenent los molts e insoportables gastos que lo Hospital General de Santa Creu de Barcelona sustenta continuament en recullir, curar, y alimentar la moltitut de malalts que de totes parts arriben a ell, y particularment als soldats que ab galeres, y altres vaxells, solen venir en gran numero" ${ }^{12}$. Con la llegada del nuevo monarca borbónico, este mismo organismo continuó siendo el principal sanatorio militar del territorio. En este sentido, la nueva dinastía siguió la estela de Carlos II, al parecer, con cierta rentabilidad para las arcas reales: "los soldados enfermos con notorio beneficio de la Real hazienda [...] cuidaba dicho hospital de toda su assistencia en lo espiritual y temporal con la sola subvencion de la racion de pan y real de este vellon por soldado"

Desde el primer momento en que la guerra aparece de forma estable y duradera en los territorios de la Corona de Aragón (agosto de 1705), en Cataluña ya se constata una situación precaria en términos sanitarios. Cuando la armada angloholandesa se presentó delante de Barcelona, parte del estamento militar que guarnecía la capital catalana sufría -o había sufrido- una afección. Según los administradores del hospital de la Santa Creu, la gran mayoría de ingresos estaban provocados por una epidemia de sarna (o roña) que afectaba a muchos habitantes de dicha plaza, entre ellos, obviamente, los militares. En 13 de julio de 1705, cuando se promulgó dicha circular, en el Hospital se contabilizaban más de 700 altas. Incluso fue necesario habilitar otros espacios - como las oficinas- para poder dar cabida a todo este grueso de afectados, ya que las estancias habilitadas para este fin no daban abasto ${ }^{14}$. Precisamente, los registros de entrada confirman este mismo hecho. Cuando el investigador aborda esta documentación constata que la cantidad de militares que ingresaron durante los meses de julio y agosto es muy elevada. Incluso se contabilizan algunos registros más en dichos meses de verano (904) que en septiembre y octubre, que es cuando tiene

10 Massons i Esplugas, op. cit., vol. I (nota 6), pp. 209-210 y 217.

11 Danón Bretos, J.: Visió històrica de l'Hospital General de la Santa Creu de Barcelona, Barcelona, Fundació Salvador Vives Casajuana, 1978, pp. 109-110. Massons i EsPlugas, op. cit. (nota 6), p. 225.

12 Biblioteca de Catalunya (BC), Arxiu de l'Hospital (AH), 617.

13 BC, AH, Caja 13, núm. 131.

14 BC, AH, 616. 
lugar el asedio aliado y, en teoría, cuando deberían incrementar las entradas en el Hospital (889) ${ }^{15}$. El dietario del futuro consejero cuarto de Barcelona, Emanuel Mas, constata esta situación, ya que dejó constancia de cómo "una roña tant apegadissa y contagiosa" afectó a los militares durante los meses veraniegos ${ }^{16}$.

Durante los meses previos a la salida del ejército borbónico de Cataluña, antes de la capitulación del virrey Velasco, los hospitales que albergaban soldados del rey eran básicamente cuatro: Barcelona, Tarragona, Girona y Roses. Precisamente éstas eran las plazas donde había guarnición permanente. Que nosotros sepamos, en Barcelona es donde se localizan los mejores fondos para estudiar esta coyuntura. De hecho, no tenemos constancia de que se haya conservado documentación del sanatorio de Girona, así como del de Tarragona. De Roses sólo conocemos la existencia de un libro sobre medicina y cirugía, fechado en $1704^{17}$. Asimismo, hacemos notar la presencia de este hospital en plena guerra de Sucesión, dado que el gobernador Juan Marquina pidió permiso para traer a un religioso de Castelló d'Empúries para atender espiritualmente a un grupo de militares enfermos, entre ellos muchos franceses ${ }^{18}$. En cualquier caso, constatamos que los cuatro hospitales fueron asistidos económicamente por la Real Hacienda, ya que hemos documentado distintas partidas para dichas iniciativas. De los presupuestos que manejaba Velasco, entre los meses de febrero y julio de 1705, el hospital de la Santa Creu de Barcelona recibió 19.200 reales de plata para la manutención de los militares albergados. Mucho menos recibieron los otros hospitales, algo evidente si tenemos en cuenta el volumen de soldados que manejaba cada edificio. Para los mismos meses contabilizados, entre los tres hospitales periféricos (Girona, Tarragona y Roses) sumaron 21.008 reales de plata ${ }^{19}$. Quedaba claro que el sistema sanitario militar borbónico -durante los primeros años del reinado de Felipe V- usaba los hospitales ya existentes en la red catalana, pagando una cuota en concepto de cura y manutención del soldado convaleciente. Actualmente, aún no sabemos con precisión la cuantía que comportaba mantener a la tropa durante estos primeros momentos de la guerra. De todas formas, extrapolando algunas informaciones de los dos ejércitos enfrentados, es muy probable que conllevara el pan de munición y una cantidad económica que oscilaría entre 1 y 5 reales por ingreso, dependiendo de la plaza que ejerciera el militar.

En cualquier caso, este modus operandi se vio truncado por la llegada de los aliados a la costa barcelonesa. Las dudas iniciales a la hora de encarar el sitio demoraron tres semanas el inicio de las maniobras de ataque. Después de un mes de enfrentamientos, las fuerzas aliadas consiguieron reducir la plaza, lo que posibilitó la entrada triunfante del archiduque Carlos. Gracias a las capitulaciones signadas entre Peterborough y el virrey Velasco, el resto del ejército borbónico se embarcó en las naves

\footnotetext{
15 BC, AH, 134, ff. 205-422.

16 Citado en Albareda i Salvadó, J.: El inicis de la Guerra de Successió a Catalunya, vol. II, Tesi doctoral, Universitat Autònoma de Barcelona, 1990, p. 383.

$17 \mathrm{BC}, \mathrm{AH}, 168$.

18 AHN, Estado, leg. 394. Carta de Juan Marquina a Grimaldo. Roses, 1 de mayo de 1710.

19 AHN, Estado, leg. 2947. Carta de Velasco a Grimaldo. Barcelona, 2 de agosto de 1705.
} 
facilitadas por el general inglés para marcharse en dirección a Almería ${ }^{20}$. Algunos oficiales y suboficiales del ejército derrotado (261 militares) se pasaron al enemigo ya que muchos de ellos enfermaron o fueron heridos. Es interesante cruzar la documentación que facilita el virrey con los registros de entrada del hospital barcelonés ya que se constata la existencia de militares que se enrolaron al ejército austriacista previo paso por el sanatorio ${ }^{21}$. Quién sabe si incluso el mismo edificio hospitalario posibilitó que asentaran plaza en dicho ejército debido a que los suboficiales y la tropa de ambos ejércitos reposaron en las mismas cámaras.

Cuadro 1. Número de entradas del estamento militar en el hospital de la Santa Creu de Barcelona (1705).

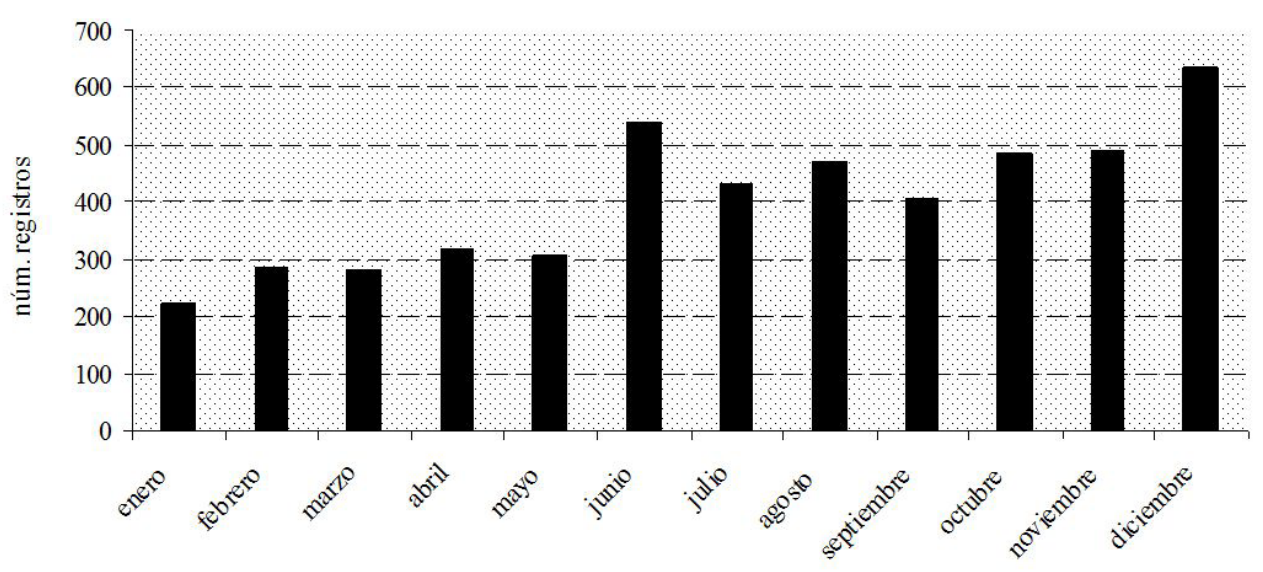

Fuente: Elaboración propia, BC, AH, 134-135.

Poco después de la expulsión del Principado catalán, las armas del duque de Anjou se centraron en recuperar nuevamente Barcelona -y Cataluña- empezando un proceso de concentración militar en la frontera oriental aragonesa, concretamente, en la línea Barbastro-Mequinenza-Maella ${ }^{22}$. En esta geografía, Fraga tuvo mucha importancia, no sólo por su situación geográfica, sino por la resistencia que ofreció a los rebeldes partidarios de la casa Habsburgo. Desde la llegada de los aliados a Cataluña, la cúpula borbónica respondió juntando sus fuerzas militares en la capital del Bajo Cinca, a pesar de que posteriormente fueron retiradas a Bujaraloz ${ }^{23}$. Este repliegue respondía a la maniobra fulminante que Felipe $\mathrm{V}$ quiso plantear ya que el ejército franco-español se abalanzó sobre la Ciudad Condal. El monarca tenía la intención de

\footnotetext{
20 AHN, Estado, leg. 664-1. Carta de Velasco a Grimaldo. Castillo de Carboneras, 23 de noviembre de 1705.

21 AHN, Estado, leg. 466. Carta de Velasco a Grimaldo. Almería, 1 de diciembre de 1705. BC, AH, 134.

22 Pérez Álvarez, M. B.: "La Guerra de Sucesión en Aragón”, Cuadernos-CEHIMO, 29 (2002), p. 83.

23 AHN, Estado, leg. 264. Para estudiar los sucesos acaecidos en Aragón durante los agitados años de 1705-1706, véase Pérez Álvarez, M. B.: Aragón durante la Guerra de Sucesión, Zaragoza, Institución “Fernando el Católico", 2010, pp. 85-136.
} 
sorprender al Archiduque con una auténtica "ofensiva relámpago" y por este motivo ignoró la opción de proteger la retaguardia ${ }^{24}$.

El 3 de abril de 1706, unos 28.000 combatientes del bando borbónico iniciaron un nuevo sitio sobre Barcelona ${ }^{25}$. El monarca -que quiso liderar la operación en persona- situó su campamento real en Sarrià, a las afueras del llano barcelonés. Desde Francia, los mariscales Tessé y Noailles también acudieron al encuentro. A través del cronista Castellví sabemos que el hospital de campaña se situó en Gracia, seguramente en el convento de dicha población, tal y como nos sugiere otra documentación ${ }^{26}$. Los resultados de la maniobra militar fueron un auténtico desastre para los intereses borbónicos y, a pesar de tener la brecha muy avanzada para asaltar la ciudad, los refuerzos marítimos aliados llegados desde Gibraltar lograron contener la embestida. Desenlace: una fuga precipitada hacia el Rosellón, dejando en el mismo campo avituallamientos, pertrechos de guerra, artillería, armas y hasta los enfermos. De nuevo, Castellví facilita algunas informaciones. El historiador austriacista apunta que fueron abandonados 1.640 soldados. De estos, 665 estaban en la villa de Gracia y fueron protegidos por una guardia que envió Peterborough -50 hombres montados- ${ }^{27}$. El dietario de la Generalitat cifra en más de 1.000 los enfermos que albergaba este edificio, sin contar los que quedaron en distintas casas diseminadas por Sarrià ${ }^{28}$. Otras fuentes barcelonesas indican que fueron más de 2.000 los militares abandonados ${ }^{29}$. La documentación inglesa relata un número sustancialmente superior en relación a los soldados enfermos y heridos. Concretamente, se constata la existencia de tres grandes hospitales con alrededor de 5.000 hombres que fueron capturados ${ }^{30}$. Estos prisioneros de guerra fueron atendidos por la Royal Navy ${ }^{31}$, lo que explicaría porqué en el registro del hospital de la Santa Creu no aparece ningún rastro de ellos. De todas formas, algunos de estos prisioneros fueron asesinados por los migueletes, como indican también las fuentes inglesas ${ }^{32}$.

Felipe V -y su ejército- volvió a Madrid a principios de junio de 1706. Durante su periplo atravesó territorio francés, viajando desde el Rosellón a Castilla pasando por el Pirineo navarro. Esta rápida marcha provocó la huida del Archiduque, que aprovechando el vacío de poder, intentó penetrar, sin éxito, en la Corona castellana. Este hecho forzó al pretendiente Habsburgo a buscar la protección de Valencia, donde se resguardó hasta el 4 de marzo de 1707, cuando emprendió el camino de retorno a Cataluña ${ }^{33}$.

24 Torras i Ribé, J. M.: "Les etapes de l'ocupació borbònica a Catalunya (1706-1713). Un debat pendent sobre el conflicte successori a Catalunya: aixecaments populars, guerra peninsular i conjuntura internacional”, Butlletí de la Societat Catalana d'Estudis Històrics, XVII (2006), p. 17.

25 Castellví, F.: Narraciones Históricas, vol. II, edición al cuidado de Mundet, J. M. y Alsina, M., Madrid, Fundación Francisco Elías de Tejada y Erasmo Pèrcopo, 1998, p. 25.

26 Ibídem, p. 117. BC, F. Bon. núms. 554 y 5723.

27 Castellví, op. cit. (nota 25), p. 117.

28 Sans i Travé, J. M. (dir.): Dietaris de la Generalitat de Catalunya, vol. X. Barcelona, p. 702.

29 BC, F. Bon. núm. 5723.

30 Gruber von Arni, E.: Hospital Care and the British Standing Army, 1660-1714, Aldershot, Ashgate, 2006, p. 162.

31 Callender, G. (ed.): The life of Sir John Leake, vol. II, Londres, 1920, pp. 46-47.

32 Gruber von Arni, op. cit. (nota 30), p. 162

33 Castellví, op. cit. (nota 25), pp. 346-347. 
Durante esta campaña de 1706 los dos reyes habían estado cerca de sus respectivos objetivos pero ninguno de los dos había conseguido consolidarlos. Llegó el invierno $\mathrm{y}$, con ello, la preparación para la campaña siguiente. A su vez, la Península Ibérica quedaba dividida en tres franjas bien diferenciadas, con la periferia (Portugal y Corona de Aragón) partidaria del rey-archiduque y el centro (Castilla) fiel a Felipe V.

En este contexto se planificó una guerra más lenta, priorizando la dominación del territorio que ocupaba cada ejército. A grandes rasgos, después de la batalla de Almansa y la consiguiente victoria borbónica ( 25 de abril 1707), el ejercito francoespañol cercó Cataluña, que era el centro de la resistencia austriacista. Con el control más o menos estable de Aragón y Valencia -al menos de las vías de comunicación con Castilla- las fuerzas militares penetraron en el Principado catalán, consiguiendo reducir Lleida a finales de 1707. La conquista de esta plaza es absolutamente fundamental para entender la política de expansión borbónica que prosiguió durante los años 1708-1713. Lleida era una ciudad cercana a la frontera y al mismo tiempo, avanzadilla desde los fortines aragoneses como Barbastro, Monzón, Mequinenza, Fraga o Alcañiz. En otras palabras, el ejército borbónico consiguió tejer una red más o menos estable que posibilitó una penetración con "garantías" en el territorio. Esta red, facilitó el suministro de toda clase de pertrechos, ya fueren para alimentar a la tropa como para la práctica del combate. En paralelo, en esta zona de "seguridad" situada en la retaguardia, se creó un entramado sanitario exclusivo para los soldados. Y estos hospitales fueron impulsados desde la iniciativa privada ya que la Corona concretó, como veremos, distintos asientos hasta la ocupación total del territorio.

Por el reglamento promulgado el 28 de julio de 1705, sabemos que los comisarios de guerra eran los encargados de velar que en los hospitales donde se recuperaban los militares borbónicos estuviera todo en orden ${ }^{34}$. Precisamente, gracias a un comisario del ejército en la frontera catalanoaragonesa -Clemente de Aguilar-, constatamos que en Lleida, en 1710, Esteban Faizones (director del hospital) era el podatario de Pedro Carlos de Leugeac ${ }^{35}$, arrendatario de los Reales Hospitales de Felipe V desde 1704 hasta $1710^{36}$. En líneas generales, dicho informe denota una falta general de utensilios, de alimentos, de medicamentos, de elementos litúrgicos y de personal sanitario. Que el mundo castrense sufriese la "falta de un todo" -como a menudo aparece en la documentación - no era una novedad relevante, más bien era la normalidad de la época. De todas formas, algunos datos del parte informativo son muy reveladores e ilustran el panorama sanitario existente en los hospitales fronterizos. Cuando se hizo dicho examen (27 y 28 de julio de 1710), la ratio de médicos y soldados atendidos era de 1 por cada 300-400. De hecho, el mismo documento informa que era imposible que un médico pudiera cumplir con sus funciones con tal volumen de enfermos y situaba el límite alrededor de los 200 diarios. En el momento de la revisión, en Lleida se contabilizaban 1.500 soldados y en Fraga, Mequinenza y Monzón se repartían otros 1.200. La falta de ayudantes en la botica también era patente y según las noticias de Clemente de Aguilar eran necesarios 28, en lugar de los 8 que había.

\footnotetext{
34 Portugués, op. cit. (nota 5), tomo X, p. 339-340.

35 AHN, Estado, leg. 758.

36 AHN, Estado, leg. 399.
} 
Lo mismo sucedía con los cirujanos practicantes, señalando que faltaban 16. Por otra parte, la comida era insuficiente y las raciones de alimento señaladas en el asiento (4 caldos diarios) no se cumplimentaban: "en que biene a tener gran útil el asentista y detrimento los enfermos" ${ }^{37}$. Además de Lleida, Pedro Carlos de Leugeac se encargó de gestionar los hospitales de Fraga, Monzón, Barbastro, Arén, Ainsa y Mequinenza. A su vez, probablemente controló otras instalaciones de España ya que gracias a un memorial de 1711 constatamos su presencia en los sanatorios de Talavera de la Reina, Guadalajara y Torija ${ }^{38}$.

Considerar que esta mala atención en los hospitales era responsabilidad del asentista no sería exacto del todo. Más bien fue un fracaso compartido. Debemos tener presente que algunos aspectos tenía que garantizarlos el rey y, a menudo, ésto o no sucedía o acaecía tarde. Éste era el caso de los nombramientos de médicos y sus respectivos sueldos. Asimismo, los aspectos relativos a la liturgia también tenía que satisfacerlos la Corona. Así, en Lleida, no había altar para administrar los sacramentos y también fue incluido como agravio. En cualquier caso parece vislumbrarse que el informe negativo tuvo cierta relevancia para que la Corona no prolongase los contratos con Pedro Carlos de Leugeac. Otros documentos también manifiestan la nula eficiencia de este director y la opinión negativa que pareció tener entre los oficiales borbónicos: "La mala orden que ha havido en los ospitales de Lérida y Fraga en Henero, Febrero, Marzo y Abril de este año, por la ninguna asistencia que los ha subministrado don Pedro Carlos de Laugeac, que ha pasado en el nombre por su Asentista los citados meses; ha hecho que se hayan muerto mas de 1.500 hombres que havian entrado en ellos de las reclutas" ${ }^{39}$. En esta misma línea, el coronel Francisco de Evoli expresaba su descontento argumentando que las pocas reclutas que habían conseguido sólo servían para reemplazar a los hombres que se perdían diariamente "por causa de la poca asistenzia de este hospital y alguna deserzion" 40 .

Seguramente nunca sabremos con exactitud cuáles eran las insuficiencias de estos sanatorios pero podemos hacernos una idea aproximada gracias a una instrucción que se divulgó con posterioridad a la ordenanza de hospitales (24 de mayo de 1708) ${ }^{41}$. Destacaremos que los enfermos tenían que estar en la cama solos, salvo un contratiempo de importancia. Para poder ingresar en el hospital, el soldado necesitaba previa certificación de su capitán, oficial o gobernador de la plaza; asimismo, cuando salía, el controlador que había recibido este boleto tenía que expedir una cédula para su reintegración a la compañía. Se tenía que elaborar un registro exacto del ingresado, donde quedara anotado el nombre, la compañía, el regimiento y el período de hospitalización. El asentista tenía que contar con todo el personal necesario para poder servir en las distintas cámaras que formaban el hospital. Una vez se cerraban las puertas, el director y el controlador estaban obligados a computar los enfermos que

37 AHN, Estado, leg. 758. Carta del marqués de Castelar al marqués de Mejorada. Lleida, 3 de agosto de 1710 .

38 Ibídem.

39 AHN, Estado, leg. 394. Carta de Miguel Fernández Duran a Grimaldo. Campo Real de Corbins, 31 de mayo de 1710 .

40 AHN, Estado, leg. 383. Carta de Francisco de Evoli a Grimaldo. Fraga, 16 de marzo de 1710.

41 Archivo General de Simancas (AGS), Secretaría de Guerra. Suplemento, leg. 269. 
albergaban. Los gobernadores tenían que visitar los hospitales dos veces a la semana como mínimo. Igualmente, uno de sus objetivos -junto al sargento mayor de la plaza y al comisario de guerra- era evitar cualquier tipo de fraude que se pudiera producir. De lo contrario, si detectaba alguna muestra de irregularidad, el controlador y el director tenían que abonar 100 doblones cada uno y eran apartados del cargo durante seis meses. Los médicos y cirujanos tenían que visitar dos veces a los convalecientes (mañana y tarde) y, sobre todo, tenían que comprobar que los soldados estuvieran verdaderamente enfermos.

Pero a partir de la campaña de 1711, la Corona no quiso seguir manteniendo negocios con el mencionado asentista y pactó nuevos contratos con Santiago Rubio de Aponte, que dirigió los hospitales hasta el final de la campaña de 1713. Este primer contrato se estableció desde el 15 de marzo de 1711 hasta el 15 de marzo de $1712^{42}$. Desde los primeros momentos del acuerdo, el arrendatario ya experimentó problemas para llevar a cabo su tarea, básicamente, a causa de la demora a la hora de recibir sus suministros. En verano de este mismo año, Aponte representó quejas a Grimaldo y al príncipe T'serclaes de Tilly, ya que necesitaba bagajes para poder transportar bizcochos, huevos, vino, 150 camas y 2.000 fanegas de harina. El contratista se justificaba en los siguientes términos; "[El asentista] a procurado sacrificar gustoso en él, [asiento] todo el caudal de su patrimonio y el de muchos amigos suios en el cumplimiento de su obligación (mediante la corta anticipazión que se le dio para esta provisión)" 43 . En juego no sólo estaba su prestigio -y su dinero- sino la de terceros que habían invertido en la sanidad militar para poder incrementar su patrimonio. Una cuestión como la de los bagajes podía ser clave y sin ellos sería aun más difícil poder abastecer a los 3.000 enfermos diarios durante los seis meses de campaña ${ }^{44}$. En un conflicto como la guerra de Sucesión, donde sobre todo en Cataluña no existió un frente claro hasta 1708 -año en el que el ejército borbónico se hizo fuerte en Tortosa, Lleida y Balaguer-, atacar a los suministros de provisiones era una de los objetivos militares tanto de los migueletes catalanes como de los voluntarios aragoneses que lucharon en el bando austriacista. Incluso en los contratos de provisión de hospitales -que abordaremos a continuación-, quedó reflejado este hecho ya que se documenta el robo de 300 medias camas en el tránsito de Cervera a Balaguer por parte de los migueletes ${ }^{45}$. El mismo príncipe T'serclaes de Tilly, máxima autoridad militar en Aragón, lo explicaba a Grimaldo y pedía que facilitasen dichos transportes para Aponte. Incluso recomendaba que para asegurar absolutamente los envíos, aprovechase los movimientos que hacía el ejército ${ }^{46}$. Este tipo de demandas fueron constantes ya que encontramos varias misivas del asentista en esta dirección, siempre pidiendo garantía y protección para sus abastos, lo cual nos muestra indirectamente la actividad bélica de este tipo

42 AHN, Estado, leg. 447.

43 AHN, Estado, leg. 397. Carta de Santiago Rubio de Aponte a Grimaldo. Zaragoza, 6 de julio de 1711.

44 Ibidem.

45 AHN, Estado, leg. 447. Carta de Santiago Rubio de Aponte a Grimaldo. Zaragoza, 18 de octubre de 1713.

46 AHN, Estado, leg. 397. Carta del príncipe T'Serclaes de Tilly a Grimaldo. Zaragoza, 5 de septiembre de 1711 . 
de milicias auxiliares ${ }^{47}$. No podemos menospreciar que, según las cuentas del mismo Aponte, la suma gastada en concepto de hospitales para la campaña de 1711 había alcanzado los 6.360 .000 reales de vellón ${ }^{48}$.

A pesar de que no conozcamos los términos de este primer asiento, podemos hacernos una buena idea del tipo de contratos que formularon la Corona y el asentista, básicamente, a través de las copias de los acuerdos correspondientes a 1712 y 1713. El segundo asiento (el de 1712) también duró un año justo (de 15 de marzo a 15 de marzo) y constaba de 36 artículos $^{49}$. El tercero (el de 1713) estuvo vigente durante siete meses, hasta el 15 de octubre del mismo año. En este caso, el asiento tenía 38 artículos $^{50}$. Esta documentación es una fuente de primer orden para el investigador que pretenda conocer las entrañas del negocio. Haciendo una lectura comparativa, nos damos cuenta que los contratos son bastante similares debido a que muchos artículos se repiten año tras año. Es deducible, por lo tanto, que durante la guerra de Sucesión la forma de consensuar estos aspectos relativos a la provisión de hospitales tuvo una cierta continuidad orgánica.

Por aquel entonces, Aponte se había convertido en el asentista del rey allí donde los combates se llevaran a cabo y -aunque no sabemos si ya lo era con anterioridad- boticario mayor del hospital de la Corte. Durante los años de guerra, Aponte se estableció en Zaragoza, desde donde controlaba las provisiones para los hospitales fronterizos; hasta consiguió - gracias al asiento- la posibilidad de ocupar la casa anexa a la suya para usarla como almacén ${ }^{51}$. En ambos acuerdos Felipe V no adelantó ninguna suma ya que la Real Hacienda estaba exhausta a causa de la guerra. Durante la campaña de 1712, los hospitales que estaban bajo su dirección eran los de Zaragoza, Monzón, Mequinenza, Tortosa, Lleida, Balaguer, Cervera, Zamora, Ciudad Rodrigo, Alcántara, Badajoz y el volante de campaña. Un año más tarde, los hospitales que gestionaba Aponte eran los mismos pero con algunas variaciones. Por una parte, Cervera fue ocupada por los aliados (verano de 1712) y los borbónicos tuvieron que desalojar la población, incluido el sanatorio. Por otra, se establecieron dos hospitales más en Aragón, concretamente en Fraga y Benabarre, a pesar de que poco después, como veremos, desaparecieron. En caso que las tropas reales penetrasen en territorio enemigo, todos los hospitales susceptibles de ser implantados también estarían a su cargo. De hecho, así sucedió en Tarragona a partir del verano de $1713,{ }^{52}$ una vez se firmó el Convenio del Hospitalet que reglamentaba la salida de las tropas imperiales de Cataluña.

Las condiciones de estos dos asientos, prácticamente, fueron las mismas. Una de las escasas diferencias fue la contabilización de los enfermos. Para las instalaciones

47 AHN, Estado, leg. 917. Carta de Santiago Rubio de Aponte a Grimaldo. Zaragoza, 16 de agosto de 1712.

48 AHN, op. cit. (nota 45).

49 Ibidem.

50 AGS, op. cit. (nota 41). Véase Riera Palmero, op. cit. (nota 7), pp. 26-29.

51 AHN, Estado, leg. 465. Carta de Santiago Rubio de Aponte a Grimaldo. Zaragoza, 1 de noviembre de 1712.

52 AHN, Estado, leg. 648. "Estado del asiento tercero de los reales hospitales de los exercitos de SM. en Aragon, Cataluña, Valencia, Extremadura, y Castilla que han estado ultimamente a cargo de don Santiago Rubio de Aponte, desde 15 de marzo de 1713 hasta 14 de octubre del mismo". 
de Aragón y Cataluña, el contrato de 1712 se computó sobre el pie de 3.000 enfermos diarios mientras que el de 1713 sobre 2.800. Para los hospitales de Extremadura y Castilla, el primer asiento se contabilizaba en base a 350 militares mientras que para el siguiente año se contemplaron 300. Quizá el acercamiento del final de la guerra explica esta leve reducción. El único que mantenía el cómputo era el hospital de campaña, levantado en base a 600 militares.

Para tratar a los soldados enfermos, heridos o gálicos, cada día los pacientes recibían 16 onzas castellanas de carne (1/3 parte de carnero y $2 / 3$ partes de vaca $)^{53}, 1$ cuartillo de vino (medida madrileña), 1,5 libras de pan de harina y si era necesario, se tenía que garantizar la equivalencia de esta ración en huevos y bizcocho. Para los oficiales, dicha porción era superior en cantidad pero estaba compuesta por los mismos alimentos; 20 onzas de carne, media azumbre de vino (medida madrileña) y 24 onzas de pan de harina. Como los soldados, esta ración también podía ser suplida por huevos y bizcochos.

El negocio de Aponte consistía, llanamente, en salvaguardar vidas. Cuantas más vidas conservaba, más dinero ingresaba. En Cataluña, Aragón y Valencia, donde el conflicto movilizó a más gente, Aponte ingresaba 4,5 reales de vellón al día por soldado enfermo, herido o gálico. Los oficiales comportaban 6,5 reales por jornada (el medio real era en concepto de cama). Si el hospital en cuestión lograba guarecer a un militar afectado con alguna especificidad gálica, Aponte era bonificado con 100 reales más. En Castilla y Extremadura, el arrendatario obtenía una cuantía inferior. El soldado equivalía a 3,5 reales al día. Los oficiales 4,5 reales. Igual que en los territorios de la Corona de Aragón, si el hospital restablecía a un enfermo del mal gálico, Aponte también era bonificado; ahora bien, en estos territorios la recompensa consistía en 75 reales a diferencia de los 100 que anteriormente hemos mencionado ${ }^{54}$. Otra fuente de ingresos podía representar el vestuario de los soldados fallecidos en el hospital. Si dichas defunciones acaecían y ningún oficial reclamaba las prendas, Aponte no estaba obligado a restituirlas. De todas formas, como nos sugieren algunos autores, estas cantidades debieron ser mínimas si tenemos en cuenta la totalidad del presupuesto manejado ${ }^{55}$.

Para llevar a cabo los propósitos mencionados, el contrato establecía otras ventajas de diversa naturaleza (privilegios). Buen ejemplo de ello era la exención de pagos que concedía el monarca para cualquier tipo de transporte, ya fueren directamente de los hospitales y los militares enfermos, de granos (bagajes), como del ganado necesario (ahorrándose los portazgos, pastos, pasos de puentes y barcas). Igualmente, tenía derecho a establecer los almacenes cerca de las instalaciones hospitalarias, así como su vivienda y la de los oficiales. Si era necesario -como ocurría en Lleida y Fragalos hospitales podían tener una piedra de molino y tenía que ser facilitada por las autoridades locales o los gobernadores de las plazas. De la misma forma, estos dirigentes estaban obligados a satisfacer la leña necesaria para las panaderías. En caso de que faltase harina, podían cogerla de los almacenes de provisión de granos, quedando

53 Dicha ración también podía constar de doce onzas de carnero diarias.

54 AGS, op. cit. (nota 41).

55 Martínez Ruiz, E.: Los Soldados del rey. Los ejércitos de la monarquía hispánica (1480-1700), San Sebastián de los Reyes, Actas, 2008, p. 1008. 
obligados a reemplazarla en veinte días. Si el arrendatario o sus oficiales eran presos por los enemigos, el rey tenía la obligación de costear su liberación, así como satisfacer el importe de todos los efectos y haciendas perdidas. En caso de no encontrar enfermeros para la manutención de los militares afectados, Aponte podía usar a dos soldados de cada batallón para este propósito. Estos "soldados-enfermeros" cobraban 45 reales de vellón al mes. Si se tenía que reparar alguna edificación destinada a albergar enfermos (casas y conventos), el arrendatario estaba obligado a adelantar el caudal de las reparaciones y, posteriormente, se le abonaba según certificación hecha por el director. Si el hospital no alojaba a más de 50 enfermos, tenían que ser transportados al sanatorio más cercano. De hecho, posiblemente los hospitales de Fraga y Benabarre fueron extinguidos por este motivo, ya que en un recuento del mes de junio de 1713 dichas instalaciones son dadas de baja ${ }^{56}$. Asimismo, Aponte y sus oficiales gozaban del fuero militar, lo que les eximía del siempre gravoso alojamiento ${ }^{57}$.

Es importante precisar que los empleados sanitarios de los hospitales que gestionó este asentista eran pagados por la Corona. A priori, el contratista no tenía que abonar su sueldo, pero probablemente - a pesar de que no tengamos evidencias documentales-, tuvo que hacer de prestamista a causa de la falta crónica de fondos para pagar los sueldos de los empleados del entramado castrense. De hecho, que Aponte anticipara alguna mercancía ajena al mundo hospitalario no era extraño ya que durante el asedio fracasado sobre Cardona (noviembre-diciembre de 1711) tuvo que suministrar ganado con un valor de 433.393 reales al proveedor de carnes Nicolás Greban ${ }^{58}$. Además, debemos tener en cuenta las grandes sumas que acarreaba gestionar este tipo de actividades. Según un cómputo realizado por el gobernador de la Real Hacienda, el obispo de Gironda -con la intención de conocer el posible desembolso que conllevarían los hospitales de Cataluña y Aragón en el asiento de 1713-, preveía una cifra de 418.740 reales de vellón mensuales. Como hemos visto, el contrato duraba siete meses, lo que se traducía en una cantidad equivalente a los 2.931 .180 reales. Los hospitales extremeños y castellanos que contemplaba el mismo asiento importaban 34.432 reales al mes (241.027 los siete meses). En otras palabras, según las previsiones del gobernador de la Real Hacienda, el arrendamiento de 1713 le costaría a la Corona 3.172.207 reales de vellón, suma que no contemplaba ni el hospital de campaña (a causa de su difícil planificación), ni los sueldos del personal médico (cirujanos, boticarios, médicos, capellanes, controladores), lo que implica que el montante tuvo que ser superior.

El hospital de campaña era el más costoso en términos monetarios y organizativos. El carácter itinerante debió ser un agravante en el funcionamiento diario de un hospital levantado tan sólo en base a 600 enfermos. La cantidad de las raciones eran las mismas que para los hospitales de las plazas. Asimismo, la cuantía que comportaban cada soldado y oficial también (4,5 y 6,5 respectivamente). El hospital contaba con ochenta acémilas exclusivas para el transporte de las 300 medias camas, los utensilios necesarios y las medicinas. Este aspecto era importante ya que en anteriores

\footnotetext{
56 AGS, op. cit. (nota 41).

57 AHN, op. cit. (nota 45).

58 AGS, op. cit. (nota 41).
} 
campañas se habían extraviado la mayor parte de ropas, fármacos y demás efectos del hospital ${ }^{59}$.

El establecimiento de hospitales militares impulsados desde la iniciativa privada se implementó únicamente cuando el frente bélico en cuestión estaba activo. Si existían combates, existían hospitales gestionados indirectamente. Posteriormente, cuando la zona se estabilizaba, el sanatorio desaparecía y los militares que necesitaban asistencia se recuperaban en las instalaciones sanitarias de las distintas localidades. En términos generales, en Cataluña, ésto sucedió en la fase final de la guerra, sobre todo a partir de septiembre de 1714. En Valencia en cambio, encontramos la otra cara de la moneda. A partir de la batalla de Almansa las fuerzas borbónicas controlaron progresivamente el territorio. En 1708, cuando Pedro Carlos de Laugeac gestionaba el asiento de hospitales, sabemos que la ciudad del Turia contaba con este tipo de instalaciones ya que hemos localizado el sueldo que cobraban los empleados de dicho sanatorio. En total, la hacienda regia tenía que abonar mensualmente 230 escudos $^{60}$. Sin embargo, cinco años más tarde, este hospital ya no existía y el asentista de turno (Santiago Rubio de Aponte), no tenía ningún tipo de obligación. Según un recuento de 1713, se puede apreciar perfectamente esta situación: "Los ofiziales y soldados que enferman en esta ciudad [Valencia] entran a curarse en el hospital general de ella con quien esta convenido y aprovado por el rey se an de pagar quatro reales al dia por el sustento y curación de cada oficial y tres reales tambien al dia por cada soldado, y por cada galico entrando a curazion mayor sesenta reales de vellon por una bez de mas del diario, y por la asistencia espiritual y temporal de medicos cirujanos capellanes y demas sirbientes se dan asimismo a este hospital 1000 reales al mes" ${ }^{61}$. En España otras ciudades tenían condiciones similares, donde el rey y la institución acordaban los términos de cada caso. Alicante, Orihuela, Cartagena, Sanlúcar de Barrameda, Jerez de la Frontera, El Puerto de Santa María y Cádiz, entre otras localidades de Andalucía, conforman algunos ejemplos ${ }^{62}$. Algo parecido debió acaecer en Zaragoza ya que hemos constatado distintas quejas de los administradores del hospital de Nuestra

59 Ibídem.

60 La plantilla estaba compuesta por el médico Jaime Sanz (50 escudos); el cirujano mayor Francisco Barriera (50 escudos); el controlador Miguel Angostura (75 escudos); el capellán Juan Ibáñez (25 escudos) y el practicante Francisco Burose (30 escudos). AHN, Estado, leg. 337.

61 AGS, op. cit. (nota 41).

62 Desde el punto de vista de la Real Hacienda, los hospitales de Orihuela, Sanlúcar de Barrameda, Jerez de la Frontera y El Puerto de Santa María no eran costos. En Alicante, el sanatorio era el de Sant Joan de Déu y se pagaba 2 reales y tres cuartillos diarios de vellón por enfermo y 60 reales por cada gálico recuperado. Para la asistencia de médicos y cirujanos se otorgaban 450 reales mensuales. En Cartagena, la Corona también había ajustado la asistencia con los padres de San Juan de Dios y las cuantías eran las mismas que en Alicante. En Cádiz, el sanatorio comportaba mensualmente 591 escudos de vellón y 2/3. La plantilla estaba formada por el veedor general Estevan Felipe Fanales (50 escudos de vellón); el presbítero Manuel Fulgencio de Estrada (40 escudos de vellón); el presbítero mayordomo Juan Manuel Fernández de Zamora y Molina (20 escudos de vellón); los licenciados Pedro de Aguilera, Pedro Hurtado de Mendoza, Carlos Lauría (jubilado) y los capellanes Agustín González y Juan González (66 escudos de vellón -13 escudos y dos reales cada uno-); el protomédico de la Armada Joseph Faraudo (80 escudos de vellón); el cirujano mayor de la Armada Ambrosio Guibebille (50 escudos de vellón); el agente Diego Fernández de la Gala (13 escudos, 6 reales y 2/3 de otro); el boticario jubilado Pedro Rebollo Guarino ( 25 escudos de vellón); el boticario activo Juan Otavio (15 escudos de vellón); el sangrador mayor Clemente Sánchez (10 escudos de vellón); un sacristán de la iglesia, dos practicantes de medicina, tres cirujanos, un oficial de botica, un despensero, dos roperos, 23 enfermeros, sirvientes, 
Señora de Gracia. Por un memorial que enviaron a Felipe V se entrevé la situación límite a la que habían llegado. La causa principal fue el gran número de enfermos, entre ellos militares, lo que provocó deudas de distinta índole. Concretamente, los administradores alertaban que si el monarca no facilitaba una "pronta y especial providencia, será imposible el poderse continuar la hospitalidad; porque mas de que con los trabajos que ocasionan las guerras, se han disminuido notablemente las limosnas, y de los seis mil pesos que tiene de renta el Hospital, apenas se cobra metad, por la calamidad de los tiempos; este año a sido muy excesivo en consumo con la concurrencia de los soldados enfermos y aunque se ha pagado algo de este gasto, no ha sido la mitad del que ha hecho el Hospital, ni aun la quarta parte de lo que corresponde al arreglamiento [...] con que por ocasion de todos estos accidentes, estará actualmente deviendo el Hospital cerca de treinta mil pesos, de celebración de Missas, cargos anuales, salarios, carne, carbon, azeyte y otros abastos" ${ }^{\prime 3}$.

Dos años más tarde, en 1710, la situación continuaba siendo insostenible. A pesar de las informaciones económicas y las recomendaciones que había hecho el conde de Gerena a Grimaldo con el objetivo de intentar salvaguardar la obra del "santo hospital" de Zaragoza ${ }^{64}$, el caso es que la situación no había hecho más que empeorar. Ya en diciembre de 1709, el rey había expedido dos decretos con la intención de minorizar la gravedad, pero quizás habían llegado demasiado tarde: "han crecido tanto los trabaxos publicos, que se abrigan a estas puertas que ya podía titubear la fee, $[\ldots]$ es inseparable en V.M. el ohír, y el remediar al afligido. Este hospital lo esta tanto que desde dos de setiembre de 1709 no cesan los regidores de hazer continuas y ardientes representaciones a los Reales Ministros [...] Pasan dos mil Personas las que experimentan esta hospitalidad [...] consúmense cada dia 54 arrobas de Pan [...] asimismo faltan 7.200 cantaros de vino. No hai carne sino hasta metad de febrero. Pasan de 500.000 reales las deudas que tiene contra sí esta Casa, donde se ha aumentado el hospital de soldados, por haberlo dispuesto así, y combenido el Marques de Castelar, de modo que por ajustado exacto balance, que se ha hecho del gasto cotidiano que oi lleva, pasa de 3.000 reales de plata. [...] ha llegado a tanto extremo la aflicción, que por no tener esta casa otro recurso, han empeñado los Regidores la propia plata de estos, con cuio producto y el de sus bolsillos, comen actualmente los enfermos" ${ }^{65}$. La lista de agravios es aun más larga pero no nos detendremos aquí en transcribir todos los términos de esta detallada documentación. Sin lugar a dudas, merecería estudio aparte. Cuando el investigador se acerca a esta documentación, quizás asombra la dureza expresada por los regidores de la institución. La situación no era para menos y los dirigentes sabían cuales eran las razones. Es por este motivo que al finalizar la carta pedían con insistencia al monarca que les pagase "cada mes los estados de los

\footnotetext{
un cocinero mayor, dos ayudantes de cocina, un aguador; cada uno de los 37 empleados importaba 6 escudos de vellón. Véase AGS, op. cit. (nota 41).

63 AHN, Estado, leg. 337. Memorial de los administradores del hospital de Nuestra Señora de Gracia a Felipe V. Zaragoza, 14 de febrero de 1708.

64 AHN, Estado, leg. 337. Carta del conde de Gerena a Grimaldo. Zaragoza, 27 de marzo de 1708.

65 AHN, Estado, leg. 383. Carta de los administradores del hospital de Nuestra Señora de Gracia a Felipe V. Zaragoza, 1 de febrero de 1710.
} 
soldados enfermos, conforme y arreglado por el Marques de Castelar en papel que firmó a 17 de Nobiembre de 1709"66.

En líneas generales, podemos constatar que las insuficiencias y los estragos vividos en términos sanitarios fueron usuales entre las fuerzas borbónicas inmersas en el conflicto sucesorio. La presencia de enfermedades fue una constante que se podía agudizar dependiendo del sitio y las condiciones existentes. Este hecho va estrechamente ligado al concepto del "síndrome de miseria". Estudios histórico-sanitarios sobre el primer cuarto del siglo XVIII nos indican esta realidad absolutamente presente entre los ejércitos de la época. Los partes médicos proporcionan buenas informaciones, siempre relacionadas con la subalimentación, el consumo de aguas contaminadas y los alimentos en mal estado. Esta coyuntura convertía a los militares en uno de los grupos de alto riesgo. Su gran movilidad les condenaba tanto a sufrir enfermedades como a propagarlas ${ }^{67}$. Quizás un extremo de esta situación se vivió en Girona, a causa del bloqueo austriacista que sufrió la ciudad durante más de ocho meses. En estas situaciones donde se cortaba la comunicación con la retaguardia -lo que limitaba el influjo de vituallas-, la guarnición tenía que sobrevivir con sus propios recursos. Una situación prolongada iba acompañada de un incremento de la pobreza, que cada vez era más extrema (léase también enfermedad). En julio de 1712 el hospital de la ciudad albergaba 300 soldados afectados, dos meses después ya eran $1.200^{68}$. Estos datos tan significativos nos demuestran que las estructuras militares colapsaron a la hora de garantizar un cierto restablecimiento. En ocasiones, la situación generada sobrepasaba los límites de la planificación, ya fuera en hospitales locales -como Girona-, o en sanatorios promovidos desde la iniciativa privada.

\section{Conclusiones}

Podemos considerar que durante la contienda sucesoria, en aquellas zonas de España donde no existía un frente abierto, los militares usaban los entramados sanitarios existentes en el territorio. Hemos visto cómo la Corona acordaba los parámetros de la manutención con los hospitales de turno, ya fueran civiles o religiosos. Aunque no haya sido objeto de estudio del presente trabajo, es deducible que estos organismos sufrieran un proceso de pauperización vertiginoso debido a la gran cantidad de convalecientes que debieron atender durante los años de combates. El hospital general de Nuestra Señora de Gracia en Zaragoza da buena cuenta de ello. Desde nuestro punto de vista sería recomendable que para conocer mejor esta coyuntura se aborda-

66 Ibídem. Cabe mencionar el interesante estudio de Fernández Doctor, que a pesar de no dedicar atención exclusiva a los años de la contienda sucesoria nos aproxima al hospital aragonés durante el siglo XVIII, demostrándonos que fue uno de los más importantes y activos de España. Véase Fernández Doctor, A.: El Hospital Real y General de Nuestra Señora de Gracia de Zaragoza en el siglo XVIII, Zaragoza, Instituto Fernando el Católico, 1987.

67 Zarzoso Orellana, A.: “¿Obligación moral o responsabilidad política? Las Autoridades borbónicas en tiempo de epidemias en la Cataluña del siglo XVIII", Revista de Historia Moderna, 17 (1998-1999), pp. 80-81.

68 AHN, Estado, leg. 426-1. Carta de Salvador Prats i Matas a Grimaldo. Perpiñán, 17 de julio de 1712. AHN, Estado, leg. 419. Carta de Pedro Rubio a Grimaldo. Perpiñán, 18 de septiembre de 1712. 
ran las fuentes documentales relativas a estos organismos de carácter local. Sólo así advertiríamos con exactitud el impacto que pudo significar el fenómeno bélico para la supervivencia de estas instituciones, y a su vez, nos acercaríamos mejor a la dinámica destructiva derivada del conflicto ${ }^{69}$.

En relación al frente catalán, hasta que las armas de Felipe V no consiguieron dominar Lleida (noviembre de 1707), los borbónicos no pudieron introducirse con ciertas garantías en el Principado. El control estable de la franja occidental catalana y la retaguardia aragonesa posibilitó crear una red hospitalaria propia. Inició entonces una guerra más gradual, lo que implicaba satisfacer a la tropa de pertrechos y servicios continuamente. La sanidad no fue ninguna excepción y, así como sucedía con todo lo relativo al aprovisionamiento (carne, granos, vestuario, armas, munición, etc.), la asistencia sanitaria se trabó con arrendamientos particulares. De esta forma, a partir de 1708 y hasta 1713 las principales plazas de la frontera (Monzón, Mequinenza, Fraga, Barbastro, Tortosa, Lleida, Balaguer) contaron con un entramado sanitario capacitado para atender a 3.000 enfermos diarios (globalmente). Estos hospitales podían cambiar de ubicación si la evolución del conflicto lo requería.

Que la Corona optará por la solución contractual privada para garantizar la asistencia sanitaria se entiende perfectamente si atendemos a los estudios sobre la financiación de la guerra de Sucesión. En la base de la explicación se encuentra la falta crónica de fondos de la hacienda regia, el necesario control de la misma y la constante escalada militar del período 1705-1714, lo que hacía aumentar el dispendio. Estos aspectos imposibilitaban practicar una gestión directa de todos los elementos relativos a la provisión. Anteriormente hemos apuntado como Jean Orry intentó encontrar financieros fuertes que costearan todo tipo de pertrechos, ya fueran franceses o españoles. Él mismo conocía las entrañas del negocio, fruto de su participación en los asientos de víveres durante la guerra de los Nueve Años. En la búsqueda de financieros, sobre todo, Orry intentaba atraer a negociantes que entablaran contratos con la Corona a largo plazo ${ }^{70}$. Recordemos que según nuestras pesquisas, los quehaceres sanitarios de la guerra de Sucesión fueron acordados con sólo dos contratistas, Pedro Carlos de Leugeac - cuyos asientos no hemos localizado-y Santiago Rubio de Aponte.

Los estudios de R. Torres reconstruyen las directrices de estas iniciativas privadas, donde los asentistas, además de aventurarse a iniciar negocios con la Corona, cuyos réditos económicos podían ser muy elevados, se sentían especialmente atraídos por la posesión del fuero militar y la posibilidad de ascender socialmente ${ }^{71}$. Hay algunos estudios muy ilustrativos, como lo demuestran las investigaciones sobre el asentista y prestamista de la reina Juan de Goyeneche ${ }^{72}$. Durante la guerra, Goyeneche y Ho-

69 En este sentido cabe mencionar los fondos existentes en el Arxiu de la Santa Creu de Barcelona, que se encuentran en la Biblioteca de Catalunya.

70 DuBET, op. cit. (nota 2), pp. 113-114 y 152-155.

71 Torres SÁnchez, R.: "Cuando las reglas del juego cambian. Mercados y privilegio en el abastecimiento del ejército español en el siglo XVIII", Revista de historia moderna: Anales de la Universidad de Alicante, 20 (2002), pp. 487-512. Véase del mismo autor la edición de un conjunto de estudios correspondientes al siglo XVIII en Capitalismo mercantil en la España del siglo XVIII. Pamplona, EUNSA, 2000.

72 AndúJar CAstillo, F.: "Juan de Goyeneche. Financiero, tesorero de la reina y mediador en la venta de cargos”, en GonzÁlez Enciso, A. (ed.): Navarros en la Monarquía española en el siglo XVIII, Pamplona, EUNSA, 2007, pp. 61-88. 
norato Leotardi suministraron granos de distinta clase a los ejércitos de las fronteras, cobrando cuantías muy sustanciales ${ }^{73}$. Otro ejemplo de debilidad económica regia, y en consecuencia, dependencia hacia lo privado, queda perfectamente ilustrado con el proceso de venalidad para acceder a la milicia. En este caso Felipe V tuvo que comerciar con su potestad real (patentes en blanco) para poder ennoblecer e incrementar numéricamente sus fuerzas armadas a un coste muy reducido ${ }^{74}$.

En 1713, Santiago Rubio de Aponte -el principal arrendatario que hemos estudiado-, aun no había percibido ninguna asignación de su contrato, tal y como se puede comprobar por el articulo XVIII del mismo. Fue a partir de 1714 y hasta 1717 cuando se embolsó las sumas gastadas, en principio, gracias a la extracción de las rentas provinciales de Palencia ${ }^{75}$. En trabajos futuros, sería deseable indagar sobre este personaje, absolutamente clave para conocer las interrelaciones entre la Corona y la sanidad militar en los albores del siglo XVIII. Lo que sí parece evidente es que -como nos sugieren las notas de J. Riera- el negocio de la sanidad militar debió ser muy lucrativo ya que, años después, un informe elaborado en Cataluña recomendaba que fuera la Real Hacienda quien organizara y administrara los aspectos relativos a la sanidad castrense, evitando así la gestión indirecta derivada de los arrendamientos ${ }^{76}$.

Aunque no contamos con documentación fiable para poder concluir con rotundidad la calidad del trato ofrecido en estos hospitales, quizá podemos percibir que a partir de 1711 (y los consiguientes contratos de Aponte) se ofrecieron mejores prestaciones a los soldados convalecientes. En los fondos de Estado relativos al período 1705-1714 (AHN), los únicos años donde los intendentes mostraron alguna queja con relación a la asistencia sanitaria, fue durante la gestión del asentista Pedro Carlos de Leugeac, lo que comportó la finalización de su negocio con la Corona. Los informes del marqués de Castelar y otros oficiales durante los meses veraniegos de 1710 evidencian la situación insostenible a la que habían llegado algunos hospitales fronterizos ${ }^{77}$. Ya hemos referido que el arrendatario no tenía que facilitar todos los componentes presentes en estas instalaciones sanitarias, la Corona tenía que poner de su parte (cuerpo médico, sueldos, etc.). Quién sabe si en comparación a Leugeac, Aponte supo suplir mejor las constantes insuficiencias del sistema de abastos castrense. Un buen testimonio parece ofrecerlo el brigadier José Vallejo, que en plena campaña de 1711 -bastante adversa desde el punto de vista felipista-, mientras criticaba abiertamente al general marqués de Villadarias, expresaba que "solo los hospitales

73 AHN, Estado, leg. 416-2. Extracto de la Contaduría General de Rentas Reales. Zaragoza, 19 de agosto de 1711; KAMEN, H.: La Guerra de Sucesión en España, 1700-1715, Barcelona, Grijalbo, 1974; AQUERRETA GonZÁlEz, S.: "La participación de los financieros nacionales en la Guerra de Sucesión: el abastecimiento de víveres al ejército", ToRrEs SÁNCHEZ, op. cit. (nota 71), pp. 273-314.

74 Andúdar Castillo, F.: El sonido del dinero. Monarquía, ejército y venalidad en el siglo XVIII, Madrid, Marcial Pons, 2004; en esta misma línea, véase del mismo autor "Vender cargos y honores. Un recurso extraordinario para la financiación de la corte de Felipe V”, en Castellano, J. L. y López-Guadalupe, M. L. (coords.): Homenaje a Antonio Domínguez Ortiz, vol. III, Madrid, 2008, pp. 89-110.

75 AHN, op. cit. (nota 52).

76 Riera Palmero, op. cit. (nota 7), pp. 31-32.

77 Véase notas 37, 39 y 40. 
están bien reglados y servidos, qual hasta aora no se vieron assí, ni es capaz de ser mexor en exercito alguno" 78 .

Cabe mencionar que la coyuntura sanitaria va estrechamente ligada a lo que muchos autores nos han indicado sobre la casuística de las bajas entre los ejércitos modernos $^{79}$. La deserción ha sido considerada una de las principales motivaciones $\mathrm{y}$, junto a ella, la enfermedad también se postula demoledora. Algunos resultados de nuestras pesquisas nos indican que en el conflicto que nos ocupa, situaciones de extrema fragilidad sanitaria comportaron un elevado número de bajas entre los cuerpos en campaña, incluso superiores a la huida del combatiente ${ }^{80}$. Investigaciones sobre la guerra de los Nueve Años apuntan precisamente en esta misma dirección, con porcentajes significativamente altos en algunos $\operatorname{casos}^{81}$. Sería recomendable seguir delimitando este impacto para concretar mejor estos aspectos absolutamente abrumadores en la cotidianidad del soldado moderno.

En los inicios del siglo XVIII aún se estaba lejos de conseguir una sanidad militar competente e igualitaria. Pero es justo recalcar que la estructura militar borbónica buscó recursos (públicos o privados) para intentar solventar este tipo de problemas. Los ejércitos que no atendían a estas cuestiones estaban condenados al fracaso ya que sufrían elevadas pérdidas humanas que no se derivaban del combate directo. En la base de esta explicación yace la precariedad crónica del sistema de avituallamientos militar, lo que comportaba que en cualquier período del año y en cualquier plaza del frente podía acaecer una tragedia humanitaria. La mala planificación de estos elementos era una rémora más de un mundo acostumbrado a moverse en los límites de la supervivencia.

\footnotetext{
78 AHN, Estado, leg. 397. Carta de José Vallejo a Grimaldo. Corella, 19 de julio de 1711.

79 Anderson, M. S.: Guerra y Sociedad en el Antiguo Régimen, 1618-1789, Madrid, Ministerio de Defensa, 1990, p. 49. Black, J.: La Europa del siglo XVIII, Madrid, Akal Ediciones, 1997, p. 379. White, op. cit. (nota 6), pp. 152-158.

80 Véase nota 68.

81 Espino López, A.: Catalunya durante el reinado de Carlos II. Politica y guerra en la frontera catalana 1679-1697, Bellaterra, Universitat Autònoma de Barcelona, 1999, pp. 219-221.
} 